\title{
Context Aware Building Energy Management System with Heterogeneous Wireless Network Architecture
}

\author{
Raja Vara Prasad Y, Rajalakshmi P \\ Department of Electrical Engineering \\ Indian Istitute of Technology Hyderabad \\ Andhra Pradesh, India 502205 \\ Email: ee10p004, raji@iith.ac.in
}

\begin{abstract}
Energy wastage in buildings is to be minimized to reduce the carbon footprint of electricity. Wireless sensor and actor networks (WSAN) have been providing solutions for effective energy management within buildings. In this paper, we present a decisive server based context aware energy management system for smart buildings through Cyber Physical System (CPS) models. A layered architecture for building energy management is proposed to enhance scalability of the system. Heterogeneous wireless network based multiple radio gateway is proposed and implemented to make the system more adaptive to different applications catering to variable data rates. A smart room test bed is deployed in the IIT Hyderabad campus, where the decisive server collects various physical parameters through sensors, and based on the context generates wireless control messages to power electronics based actuators. Integrating context awareness into the system increases the efficiency in terms of energy savings and was observed to be significant, around $30 \%$. The paper also presents a detailed analysis on the turnaround time required to realise the real saving after recovering investments. Applications are developed to integrate smart phones and tabloids providing web enablement to the end user. In this paper, each of the sensors and actuators in the smart room are associated with a state machine, which enables modelling of the system using Hybrid automata for future scope of applications.
\end{abstract}

Keywords-Cyber Physical Systems, context awareness, cognitive server, building energy management, heterogeneous wireless networks, turnaround time, state machines

\section{INTRODUCTION}

The ever growing energy demand needs has to be thoroughly reviewed to reduce the greenhouse gas effects and climatic changes. U.S. Department of energy reports 39 percent of total energy consumption by buildings [1]. The energy consumption of the buildings is contributing to 8 percent of total emissions. Information and communication technologies (ICT) play a key tool to support smart energy management within buildings thereby reducing the carbon footprint by at least 15 percent according to smart2020 report [2].

Research in the field of home/building energy management systems (HEMS/BEMS) is discussed in [3][5]. Adding a new device to the existing system is difficult in above mentioned systems. HEMS/BEMS should be adaptable for future integration of devices. Heterogeneous network implementation of BEMS/HEMS will provide this flexibility of adding new devices adaptively and able to communicate with each other for information. Available Short-range heterogeneous wireless networks can support interaction between machines/appliances, sensors and controlling actuators. These networks can be classified as Cyber Pysical Systems (CPS), which can serve many applications such as industry automation, smart homes, health care, environmental monitoring etc. BEMS/HEMS with heterogeneous wireless networks enable us to create new ubiquitous services for a more convenient lifestyle.

The energy management systems proposed in the literature is discussed here. Power line communication with modem and couplers for the home energy management and control system discussed in [6]. The interactions between the networked smart appliances are discussed in [7] where the services are categorized as communication power control and multimedia service with an IP. The concept of smart appliance, smart meter, and smart server is introduced in [8]. Wireless CAN protocol is proposed for home electrical appliances monitor and control in [9]. All the proposed technologies mentioned above are mostly PLC with disadvantages of low bandwidth, high initial cost, more installation time and interference from the electrical network and the electrical devices connected [10]. Full smart home automation design and implementation is discussed with Zigbee as the network in [11], [12] and [13]. 6 LOWPAN, IPV6 based home automation system is proposed and partially implemented along with 802.15 .4 nodes in [14]. Most of the above proposed technologies discussed cannot handle high data rate applications. The focus of the previous methodologies is more on the user comfort than the energy management. It's difficult to maintain Quality of service (QoS) for a wide variety of applications with a homogeneous network architecture.

PUCC P2P based heterogeneous wireless network for HEMS/BEMS is proposed in [15]. P2P nodes and sensor gateways get access to the connected network adaptively through heterogeneous network platform. A test bed of PLC and ZigBee is experimented in 2 office buildings. Though the work mentioned is suitable for BEMS still it requires many interfacing modules to connect communication gateways and electrical devices. The cost of installation increases exponentially with the number of interfacing devices.

Number of proposed standards to describe devices and enable their integration is discussed in [16]. IEEE 1451 [17] is the mostly used standard for connecting devices to microprocessors, instrumentation systems, and control/field networks. IEEE 1451 is a collection of open, common, networkindependent communication interfaces. SensorML is another standard [18] used deriving higher-level information from ob- 
servations by creating measurement models using sensors and instructions. OSGi enabled technology that enables interfacing with hardware devices using Java is discussed in Device Kit [19], and SODA [20]. The above standard which uses java is similar to our proposed gateway based architecture. Java is used for devices when hardware specific information is unknown. Although there are many similar technologies for integrating multi-vendor devices [3][5] and [15], we developed a java based server which can easily adapt to most of gateways. Details of packet structure and input port are required at the stage of integration to the server. Data collected from sensors are organized into database and retrieved by decision algorithm to generate control actions.

In this paper a new approach to building energy management systems (BEMS) is introduced with total heterogeneous wireless networks. Although Existing wired based BEMS are well established, it is difficult to wire the existing buildings in terms of cost and time of implementation. We propose a rule based server energy management system for the smart building, where energy savings is prioritized through context awareness. Decision server is independent of communication protocols used which makes it adaptable to old as well as new technologies/devices. In this work, wireless communication systems such as Zigbee, Wi-Fi, and RFID are considered which connects to the server through their respective gateways thereby making adaptive to any protocol or market available product. The proposed system also provides a lot of flexibility/comfort to end user. Web enabled energy management for local and remote operation with smart phones, tabloids, laptops etc. is another major advantage with the proposed heterogeneous architecture. Testbed was run with proposed architecture and significant energy savings are observed from the observations.

The rest of the paper is organized as follows. Section II presents the proposed architecture and section III discusses the layer wise description of system architecture for smart home. Experimental results observed from the implementation are presented in section IV. Section V concludes the paper with future work.

\section{SYSTEM ARCHITECTURE}

The proposed architecture consists of four layers as shown in Fig.1. Where each layer performs unique function. The four layers of the smart home architecture are

1. Sensor layer

2. Mote layer

3. Server layer

4. Control layer

Layered architecture is adopted so as to incorporate scalability to the system. The design and implementation of these layers is further discussed in detail in the following section.

- Sensor layer integrates various physical parameters into the system. sensors for motion, temperature, light intensity, power monitoring etc. are integrated into the architecture.

- Mote layer communicates the sensed information to the server through Zigbee and Wi-Fi network.

- Server layer aggregates all the sensed information, runs a rule based decisive algorithm, and sends control commands to the control layer based on the context.

- Control layer consists of various power electronics based actuators, which controls the loads based on the command received from the server layer.

\section{LAYER WISE DESCRIPTION OF SYSTEM ARCHITECTURE FOR SMART HOME}

Each layer of the proposed architecture constitutes of different hardware and software modules. The functionality, design and implementation of different modules in these layers are described in this section.

\section{A. Sensor Layer:}

The sensors used in the system are magnetic door status detectors, PIR sensors, RFID, Light intensity and power monitoring as shown in Fig.2. Sensors collect the physical information required to identify context. Functionality and implementation of each senor is explained through state diagrams.

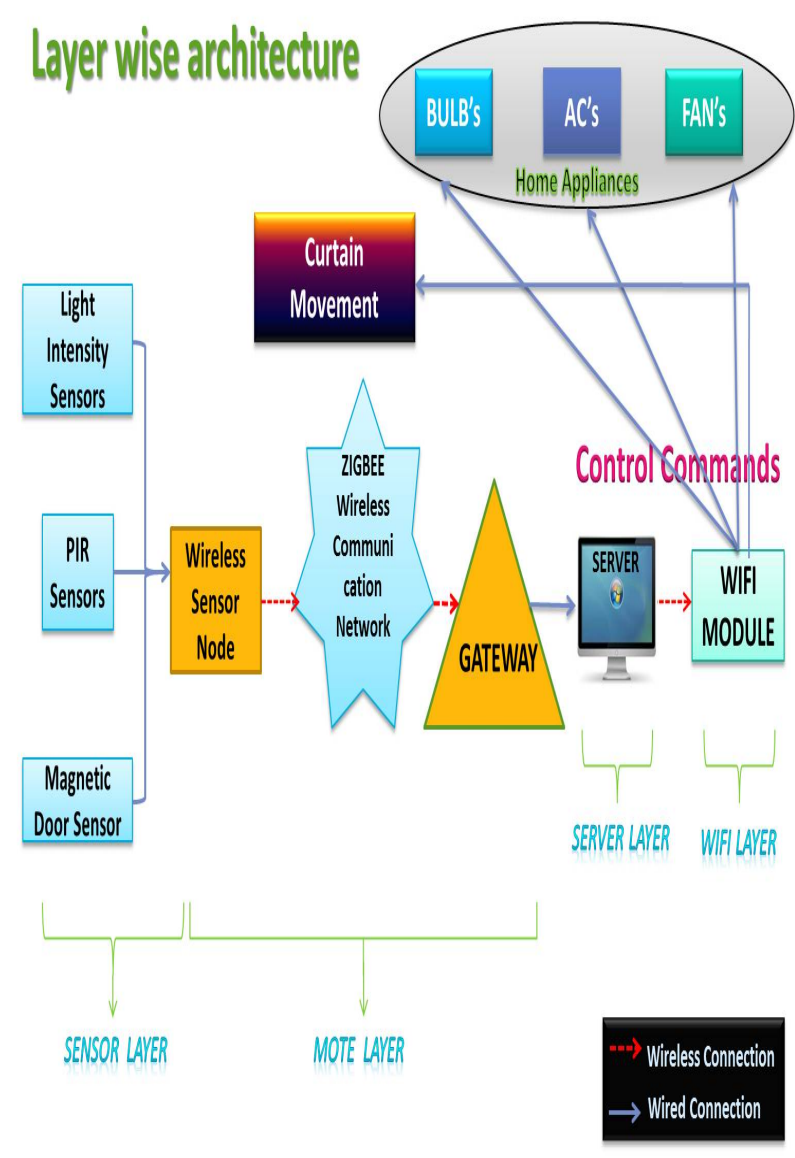

Fig.1. Proposed layered architecture for smart room 


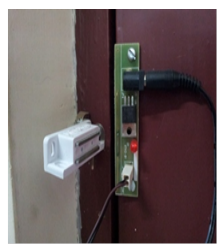

a

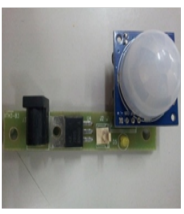

b

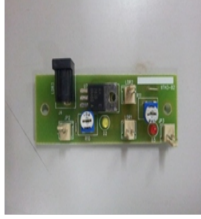

C

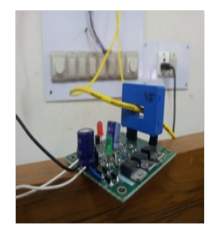

e

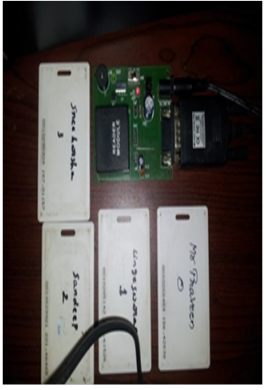

d
Fig.2. Different sensors in the smart room test bed
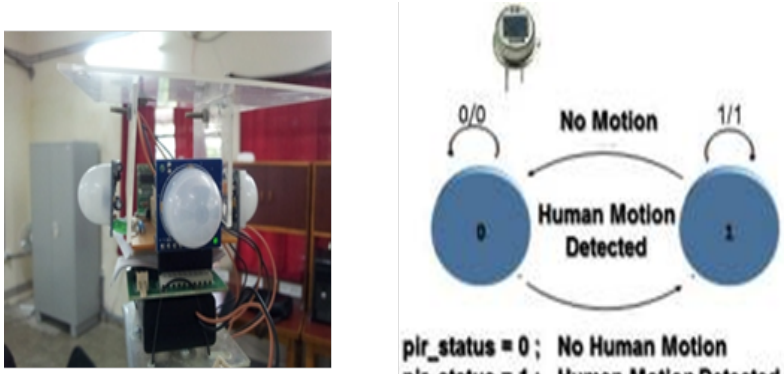

pir_status = 0; No Human Motion pir_status = 1; Human Motion Detected.

Fig.3. PIR sensor state flow diagram

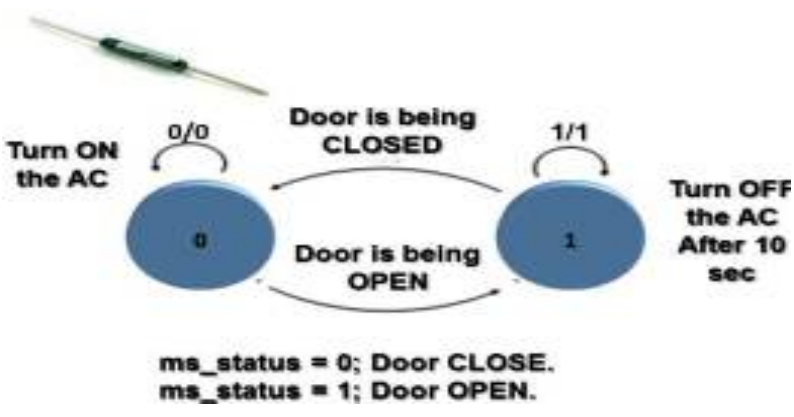

Fig.4. Magnetic sensor state flow diagram

1) PIR and RFID:: The PIR sensors (Fig.2 (b)) are targeted to detect the motion of the person within the region. RFID (Fig.2 (d)) helps keep a count on the number of people within the smart room. PIR sensor detects motion of person in angular region of 90 degree up to 4 meters. Four PIR sensors are arranged together on single pole to cover the 360 degrees of the room as shown in the Fig.3 (a). Each PIR sensor is responsible for switching on the electrical loads of its detection region. The state flow of the PIR sensor is as shown in the Fig.3 (b)

2) Power monitoring module:: Power monitoring module (Fig.2 (e)) periodically senses the power consumed by any electrical device/room/building using an energy metering IC ADE7757 [21] and signal conditioning circuit. Electrical loads which have different modes of operation are monitored to adapt future smart grid applications like priority load on/off.

3) Door Status Detector:: Door/window open or close is detected to prevent the overloading of air conditioners/room heaters in a room. Door open/close status is detected using a magnetic door sensor (Fig.2 (a)). The state flow of the magnetic door sensor is as shown in the Fig. 4.

4) Light Intensity Sensors:: Light Intensity Sensors (Fig.2(c)) detect the amount of daylight intensity. Before switching on the lights within the smart room, light intensity inside and outside the room are compared with detection of change in LDR resistance. The change in resistance is converted to lux units using calibration equation and a threshold $(\geq 500)$ on lux is considered as good to open/close the curtains. The functionality is described using the state diagram as shown in Fig.5.

5) Temperature Sensor:: Temperature Sensor is to measure room temperature to switch between fan and air conditioner according to the temperature inside the room as shown in Fig.6.

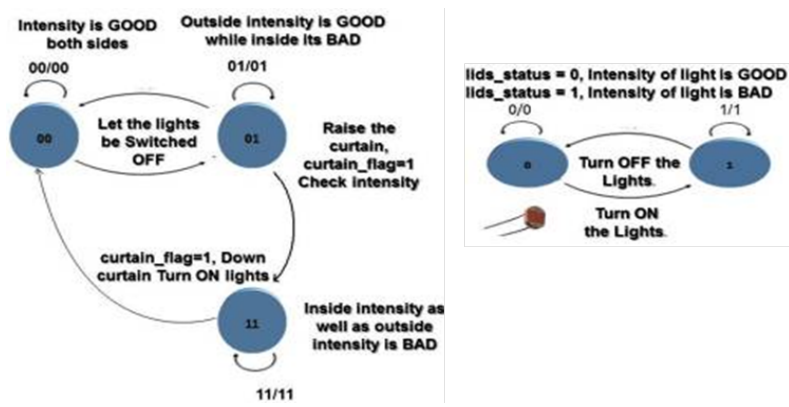

Fig.5.Light Intensity Detection Sensor state diagram

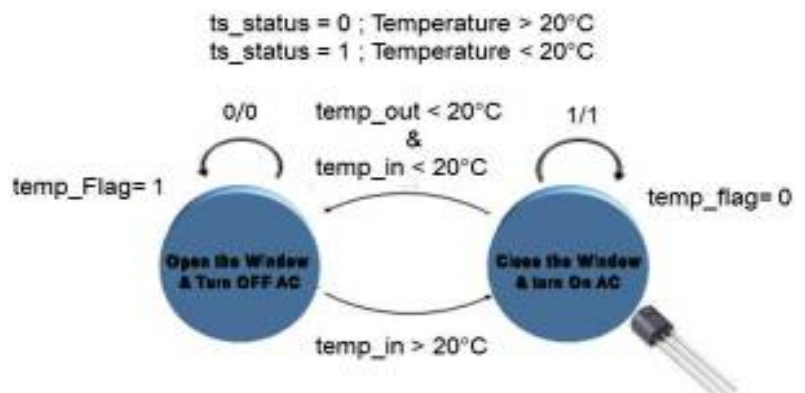

Fig.6. Temperature sensor state flow diagram

\section{B. Mote Layer:}

Heterogeneous wireless technologies are used in the test bed keeping in mind of future wireless applications of smart building, smart grid, and other wireless automated applications. Heterogeneous wireless technologies like Wi-Fi, ZigBee and GSM are integrated to server. Zigbee nodes are used to collect sensor data to server because of its proven capabilities of low power multi-hop networking. Wi-Fi is used to decode control commands from server for multiple loads. It can be used as data aggregator for total building to upload data to the internet thereby providing remote access of home energy management system. One Wi-Fi node as data concentrator of total building status can be used as part of Home Area Network (HAN) as shown in the Fig.7 which is proposed in [22]

Different application requirements like bandwidth, delay, and reliability can be met with Heterogeneous network implementation. Wi-Fi (Fig. 8(a)) communication is intended for high data rate applications like data aggregation from all local Wi-Fi nodes of building and as well as fast decode of control commands for actuation systems. Distant user can send 
control commands to the system using GSM mobile where a GSM module connected to sever will receive and forward to decision algorithm. Remaining sensors like door status detectors, temperature, humidity, light sensors are connected to reliable low power and data rate ZigBee network (Fig. 8(b)).

\section{Server Layer :}

Server layer is a java based decisive algorithm as shown in Fig.9. Which is connected to different communication gateways. Server is connected to Zigbee, Wi-Fi, and RFID gateways through different serial ports. Server receives sensor data from Zigbee, RFID, Wi-Fi and Control options from WiFi. Server is connected to Wi-Fi gateway to send the control commands to embedded WI-FI board. Electrical appliances receives control sequences from the WI-FI board. Server logs each PIR, RFID, and door sensors status from Zigbee gateways to run the decisive algorithm depending on the sensors data to satisfy the different contexts. The integrated power monitoring module is also connected to Wi-Fi module so that reading can be sent to sever for decision.

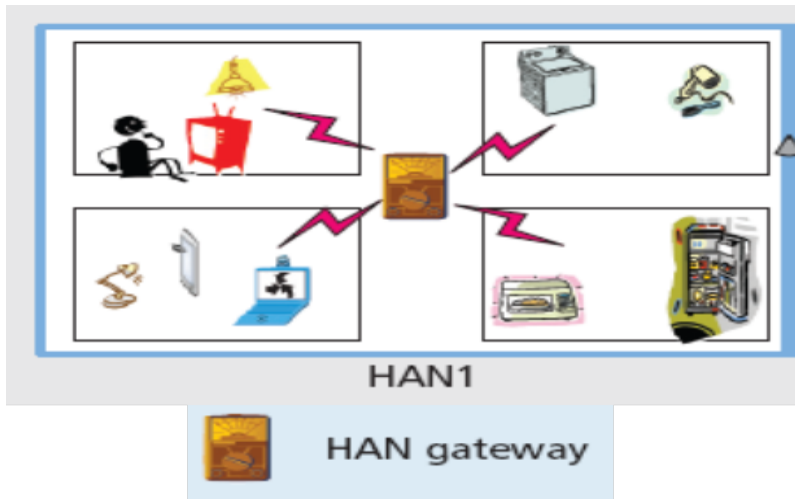

Fig.7. Home area network architecture with Wi-Fi as gateway.
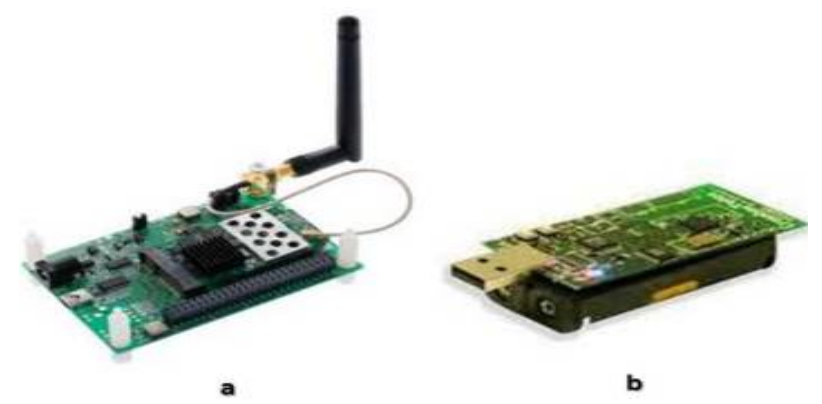

Fig.8. Communication modules used in mote layer

Flowchart of the decisive engine in the server is shown in Fig.9. The data received from the different gateways is organized into a database and an intelligent decision algorithm is run according to the context and control actions are sent to actuators. Various context for the smart building are personal occupancy, door open/close, optimal switching of lighting load with window gliders to use natural light, temperature based cooling/ heating equipment operation, real time power monitoring for real time energy management applications like smart grids. Automation of electrical loads based on context data helps in achieving energy saving.

\section{Control Layer:}

Control layer is collection of embedded and electrical actuation systems. WI-FI board is used as embedded board to decode the received control commands from server's Wi-FI gateway. Depending on the actuation signals, power electronic based actuation systems control electrical loads accordingly. Different ratings and types of actuation systems are designed for loads like light, fan, air conditioner, curtain glider/operator etc as shown in Fig.10

Having given functional description of all the layers in the smart room testbed, we now discuss experimental results observed from the testbed, the cost analysis and turnaround time in the next subsection.
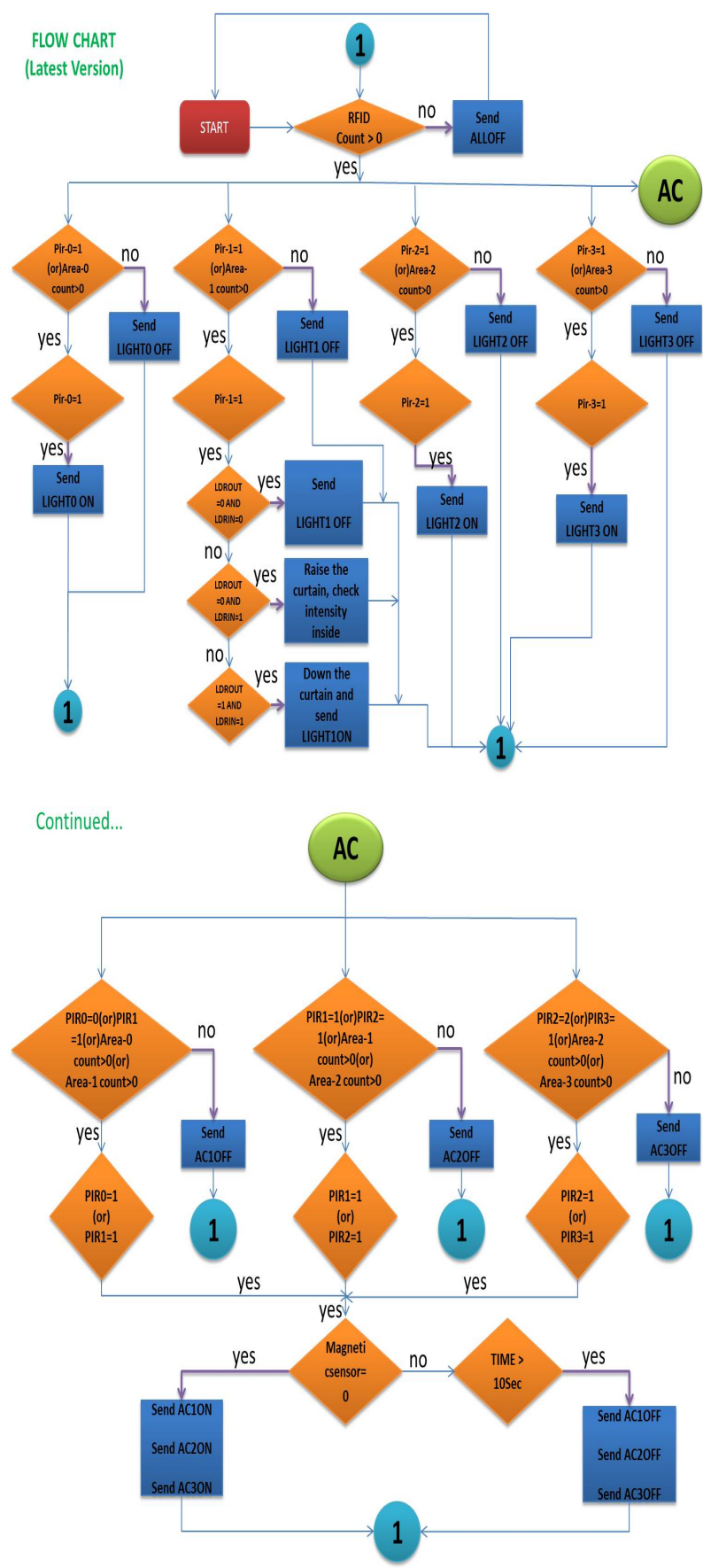

Fig.9. Decision algorithm working flow diagram 


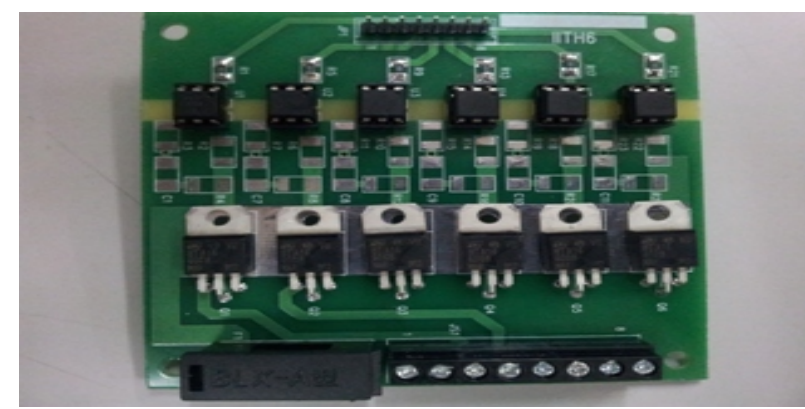

Fig.10. Power electronics based control modules

\section{EXPERIMENTAL RESULTS FROM SMART ROOM TEST BED}

Experimental details of the test bed are discussed in this section. This section is further divided into three subsections as below

a.Energy savings from the smart room testbed

b.Cost analysis and turnaround time computation.

c.Web based GUI and applications for smart gadgets (Smart phone and Tabloid)

\section{A. Energy savings from the smart room testbed}

A sample testbed is implemented in Cyber Physical Systems (CPS) lab of IIT Hyderabad with our proposed architecture. The testbed was run for 3 days with proposed system and next 3 days without proposed system. All events were logged into server for 6 days. The data collected includes sensors status, loads status, lab occupancy and power consumption details. Energy savings due to proposed architecture is analysed from the test bed results. Test bed implementation of CPS lab is explained in detail as below.

Proposed architecture is implemented in IIT Hyderabad Cyber Physical Systems (CPS) lab. Most of the required hardware and software is developed indigeneously for the test bed CPS lab. The test bed CPS lab is $30 \mathrm{X} 30$ feet room. WI-FI and zigBee networks in the present test bed are in star topology. Proposed system can be easily extendable to total building with WI-FI and ZigBee adhoc mesh networks.

Number of occupants : 8

Number of lights : 16

Number of air conditioners : 3

Number of personal computers : 8

The wattage readings of loads and their total wattage are as follows

Light : 30 watts

Total : $30 * 16=480$ watts

Air conditioners : 1600 watts

Total $: 1600 * 3=4800$ watts

Personal computers : 90 watts

Total : $90 * 8=720$ watts

Total wattage of the room is 6000 watts.

The wattage of the loads are taken from the manufacturers specifications with a $\pm 5 \%$ accuracy. The layout of testbed deployment is a shown in the Fig.11. Total lab is divided into 4 parts. Each section of the test bed is having a PIR sensor to detect motion of the occupants in its area. Loads are distributed in four parts of the room as shown in the Fig.11. where L-* stands for lights and AC-* for Air conditioner

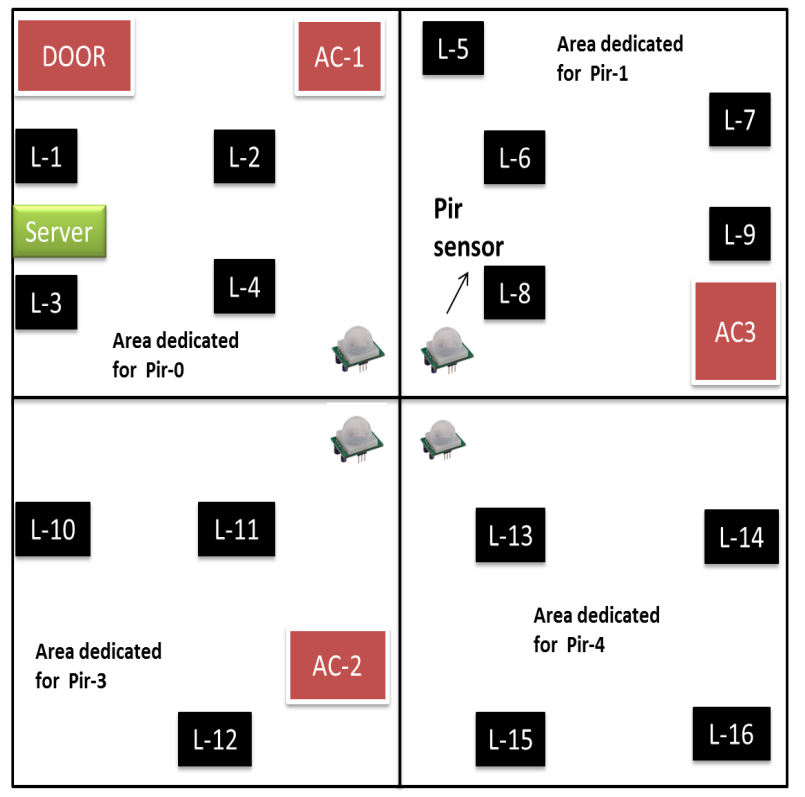

Fig.11. CPS lab layout for testbed

Each person occupying CPS lab is given a unique RFID card. Every entry or exit of occupants is logged into database of the system thereby maintaining count of persons in the lab. PIR sensor connected to a ZigBee node detects motion of the person in that area and sends this information to ZigBee gateway. Temperature sensor and light intensity sensors data is also carried out to server through ZigBee nodes. Server collects data from all gateway and runs a java based decisive algorithm to generate control actions for the actuation systems. Optimal switching of load is carried out by considering different contexts in the lab. Contexts are identified by the server from the information collected from all sensors. Some of the context are as mentioned below.

Loads are switched on only when there is motion in the coverage area of a PIR sensor. This will reduce the unnecessary wastage of energy by switching off lighting load when occupants are absent in that area. If light intensity from sun is enough for working in the lab, lights can be switched off. Light intensity sensors can be used to detect the ambient light in the room to switch on/off the lights and automate the curtains accordingly. Temperature sensor readings can be used to switch between the fan or AC. Temperature context is also used to switch on/off number of AC's according to the temperature inside the room. For example if the room temperature is below 18 degrees switch off one AC to reduce the energy consumption. Several other contexts can be incorporated to make the system more energy efficient. All the above contexts are implemented in the test bed to observe the energy savings with the proposed system.

Experimentation is carried for total of 6 days. All events for 6 days are logged in the database. occupancy of the lab and the power usage according to the no of people is as shown in 
the Fig.12. The power usage of the lab is following the pattern of increasing/decreasing with number of people present in the lab. The energy savings of the lab is given as below.

Energy savings of CPS lab for three different days with the proposed system are $28.6 \%, 19.94 \%$ and $37.13 \%$ respectively. Average savings in the energy is $28.55 \%$. Person occupancy in the lab is not same for first 3 days of experiment. All loads are switched on irrespective of occupants are present in the lab or not for the case of without proposed system. Energy is calculated by taking maximum connected load of 6000 watts for without proposed system is a valid assumption for most of the buildings.

\section{B. Cost analysis and Turnaround time calculations}

Cost involved in implementing the smart room test bed is given in Table 1.

Turnaround time which is the time after which actual saving is realized taking into account the cost of the system is as shown in Table 2. Cost of energy is assumed to be 16.5 cents/unit (Indian Scenario). The table assumes the values from previous subsection.

The testbed results shows that approximately $30 \%$ of energy savings. From the Table 2 with $30 \%$ savings of energy the turnaround time is around 239 days.

The analysis carried out in the above subsections clearly shows that our proposed building energy management system provides not only good amount of energy savings, but also cost effective solution with minimum turnaround times. Increased in volume of hardware production will still reduce the initial cost of the system.

Table 1.cost involved for the test bed implementation for smart lab at IITH

\begin{tabular}{|l|l|l|l|}
\hline sensors & Quantity & Price(USD) & Total(USD) \\
\hline PIR & 4 & 5.5 & 22 \\
\hline RFID door & 1 & 16 & 16 \\
\hline $\begin{array}{l}\text { Magnetic } \\
\text { sensor }\end{array}$ & 1 & 2 & 2 \\
\hline $\begin{array}{l}\text { Power } \\
\text { monitoring } \\
\text { module }\end{array}$ & 1 & 30 & 30 \\
\hline Control module & 15 loads & 20 & 20 \\
\hline Wi-Fi module & 1 & 150 & 150 \\
\hline $\begin{array}{l}\text { Zigbee IITH } \\
\text { node }\end{array}$ & 1 & 120 & 120 \\
\hline \multicolumn{2}{|c|}{} & total & 360 \\
\hline
\end{tabular}

Recovery of the investment depending on the percentage of savings in energy is as shown in the table

Table 2.Recovery of the investment depending on the percentage of savings in energy

\begin{tabular}{|l|l|l|l|}
\hline $\begin{array}{l}\text { percentage } \\
\text { savings }\end{array}$ & $\begin{array}{l}\text { savings in } \\
\text { units }\end{array}$ & $\begin{array}{l}\text { savings in } \\
\text { USD/day }\end{array}$ & $\begin{array}{l}\text { Recovery } \\
\text { in no of } \\
\text { days }\end{array}$ \\
\hline 10 & 3.072 & 0.5026 & 716 \\
\hline 20 & 6.144 & 1.0052 & 358 \\
\hline 30 & 9.216 & 1.5078 & 239 \\
\hline 40 & 12.288 & 2.0104 & 179 \\
\hline
\end{tabular}

\section{Web based GUI and applications for smart gadgets}

Status of all sensors, electrical loads and actuators are logged into the database in real time. To better visualize the online data of the system a web based GUI is designed as shown in the Fig.13. User with proper login details can view status of sensors, electrical loads and statistics of parameters of interest as shown in the Fig.12. User who doesnt want automation can give a manual command for controlling the loads. Up gradation of existing GUI is under process to present data in a more meaningful way to the end user.

Application for smart phones and tablets are also developed to visualize the data and control the loads as shown in the Fig.14. These applications allows user to monitor data and control loads remotely. The above applications are also for proper energy management and made adaptable to future smart grid applications.

\section{CONCLUSION AND FUtURE SCOPE OF THE WORK}

Optimal energy usage in buildings can be achieved with the help of networked wireless sensor and actuator systems. In this paper, context aware building energy management system for smart buildings is proposed with heterogeneous wireless network based sensors and actuators system. Smart building architecture proposed consists of layered structure, such as sensor, communication, server and control layers with unique functionalities. A smart room test bed was created with heterogeneous wireless environment, such as Zigbee, Wi-Fi, and GSM. Depending on the various physical parameters sensed at the sensor layer, decision engine generates the control messages to the actuators connected to the loads. The experimental results from the test bed shows that there is significant energy saving of about $30 \%$ with the proposed system in place. Further the cost analysis and turnaround time computations shows that the proposed system is cost effective with minimum turnaround duration. Applications developed for smart phone and tablet PCs gives the end user more access/control and in turn comfort. Web based GUI developed is really useful for monitoring energy usage of the building/home for both local and remote operation of electrical loads.

The future scope of the work is to deploy the system in various scenarios such as domestic, commercial offices and auditoriums. Modeling of the adaptive system with previous logged data like personal occupancy behaviour, temperature, and context is also part of future work for this project. The integration of renewable energy sources to the system is also a challenge. The smart home developed can be part of smart grid architecture which can be further researched for quality of service parameters. A complete hybrid automata model for total system is also future scope of research.

\section{ACKNOWLEDGMENT}

This work is funded by CPS project, Department of Information Technology (DIT), India. We also sincerely thank our Project staff CP.Srinivasu, Ruchi Tripathi and V.Anvesh for making this test bed implementation successful. 


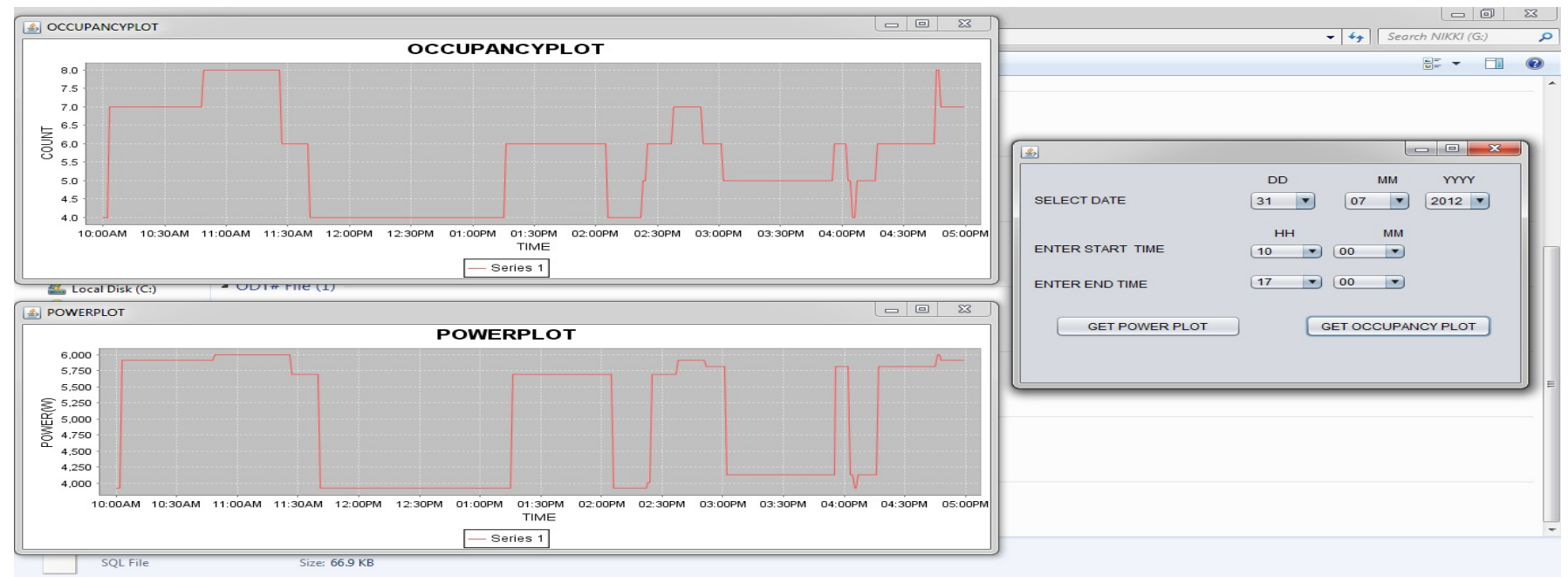

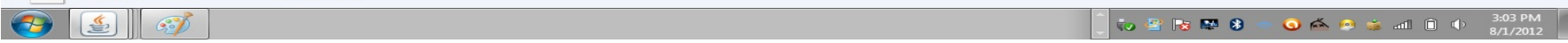

Fig.12. Power usage plot for 1 day of the experimental run

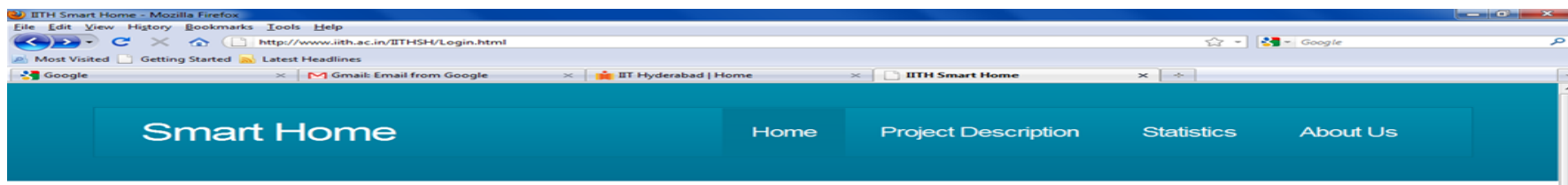

Smart Home Implementation by

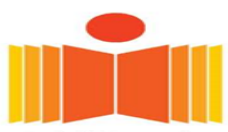

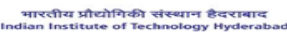

Enter Authentication Credentials

Login

[submit] Reset

Fig.13. Online web based GUI for smart room test bed
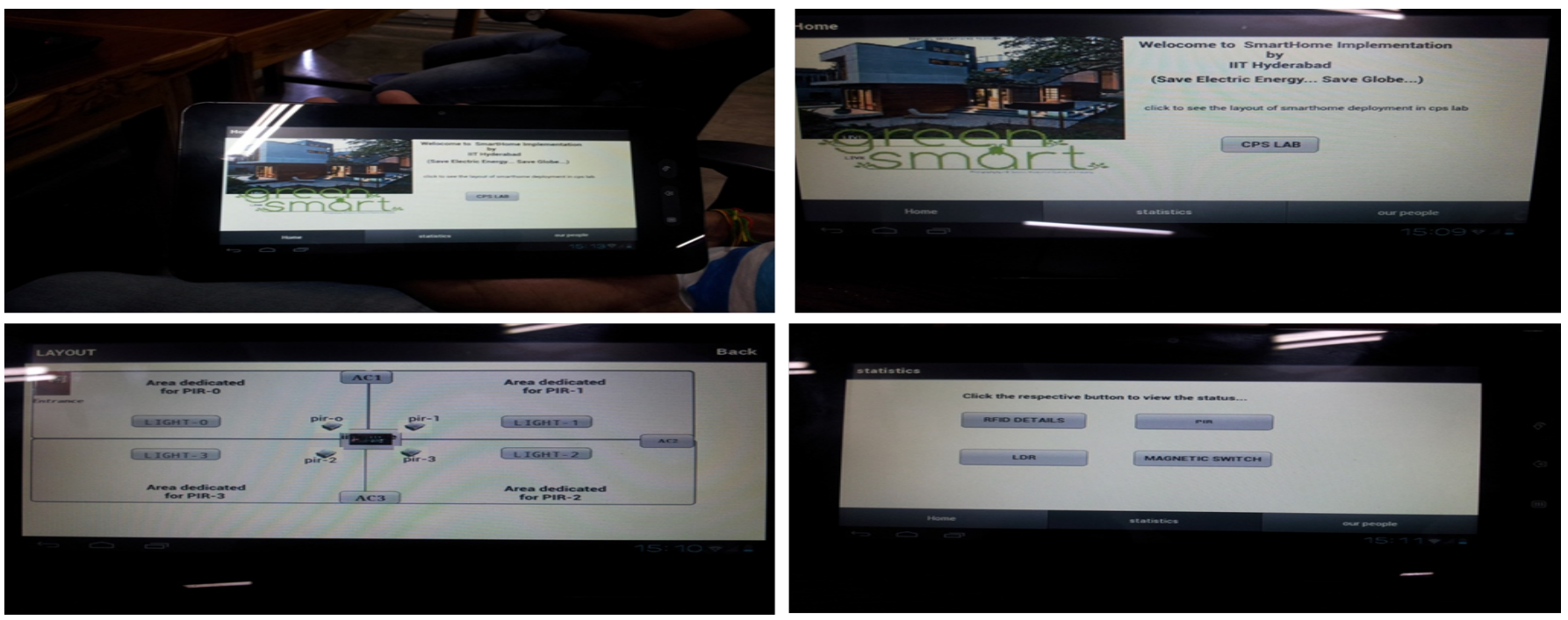

Fig.14.Tabloid application for test bed Energy management 


\section{REFERENCES}

[1] U.S. Department of Energy, Buildings energy data book.http:1/buildingsdatabook.eren.doe.gov/, 2009.

[2] SMART 2020: "Enabling the low carbon economy in the information age".

[3] L.Cao,J.Tian,D.Zhang, "Networked Remote Meter-Reading System Based on Wireless Communication Technology", IEEE International Conference on Information Acquisition, pp. 172176, 2006.

[4] M. Inoue, T. Higuma, Y. Ito, H. Kubota, "Network Architecture for Home Energy Management System”, IEEE Transactions on Consumer Electronics, Vol. 49, pp. 606613, 2003.

[5] N. Kushiro, S. Suzuki, M. Nakata, H. Takahara and M. Inoue, "Integrated Residential Gateway Controller for Home Energy Management System", IEEE Transactions on Consumer Electronics, Vol. 49, No. 3, pp. 629636 , 2003.

[6] Sheroz Khan, Rafiqul Islam,Dr Othman 0 KhalifaJanaluddin Omar, Abubkar Hassan, Ismail Adam, "Communication system for controlling smart appliances using power line communication" 0-7803-95212/06/2006 IEEE.

[7] Mario Kolberg, Evan H. Magill, and Michael Wilson, University of Stirling "Compatibility Issues between Services Supporting Networked Appliances" IEEE Communications Magazine November 2003

[8] J.J. Jamiana'M.W. Mustafa a, H. Mokhlis b, M.A. Baharudina "Conceptual Data Management and Communication for Smart Distribution System" 2011 IEEE First Conference on Clean Energy and Technology CET

[9] Wei Lun Ng, Chee Kyun Ng, Nor Kamariah Noordin, Fakhrul Zaman Rokhani and Borhanuddin Mohd. Ali "Home appliances controller using wireless controller area network (wcan) system" International Conference on Computer and Communication Engineering (ICCCE 2010), 1113 May 2010, Kuala Lumpur, Malaysia.

[10] Anatory, J., M.M. Kissaka, and N.H. Mvungi. "Powerline Communications: The Effects of Branches on Network Performance" . Power Line Communications and Its Applications, 2006 IEEE International Symposium on . 2006.

[11] Dae-Man Han and Jae-Hyun Lim "Design and Implementation of Smart Home Energy Management Systems based on ZigBee" 0098 3063/10/ 2010 IEEE

[12] Yu-Ping Tsou, Jun-Wei Hsieh, Cheng-Ting Lin, Chun-Yu Chen "Building a Remote Supervisory Control Network System for Smart HomeApplications" 2006 IEEE International Conference on Systems, Man, and Cybernetics October 8-11, 2006, Taipei, Taiwan.

[13] Alan Marchiori "Enabling distributed building control with wireless sensor networks" 2011 IEEE

[14] Bernd Michael Drge, Thomas Scheffler "Using IPv6 and 6LoWPAN for Home Automation Networks" 2011 IEEE International Conference on Consumer Electronics - Berlin (ICCE-Berlin)

[15] Hiroshi Mineno, Yuichiro Kato, Kenji Obata, Hiroshi Kuriyama, Keiichi Abe, Norihiro Ishikawa and Tadanori Mizuno "Adaptive Home/Building Energy Management System Using Heterogeneous Sensor/Actuator Networks" IEEE CCNC 2010 proceedings, IEEE Communications Society

[16] C. Chen and A. Helal, "Sifting through the Jungle of Sensor Standards", IEEE Pervasive Computing Magazine, Vol. 7, No. 4, 2008.

[17] IEEE 1451 Standard, http://ieee1451.nist.gov.

[18] SensorModelLanguage www.opengeospatial.org/standards/sensorml.

[19] Device Kit Website, www.eclipse.org/ohf/components/soda/index.php.

[20] C. Chen, A. Helal, "Device Integration in SODA using the Device Description Language", IEEE International Symposium on Applications and the Internet (SAINT), 2009.

[21] Datasheet of ADE7757 (single phase energy metering IC)

[22] Zubair Md. Fadlullah, Mostafa M. Fouda, and Nei Kato, Akira Takeuchi, Noboru Iwasaki, and Yousuke Nozaki "Toward Intelligent Machine-to-Machine Communications in Smart Grid" IEEE Communications Magazine April 2011. 